\title{
LIST OF DONORS
}

TO THE

\section{LIBRARY OF THE LINNEAN SOCIETY.}

With References to the Numbers affixed in the foregoing Catalogue to the Books presented by them respectively.

THE Royal Society of London, 438.

The Royal Society of Edinburgh, 527 .

The Asiatick Society, 528.

The Society for the Encouragement of Arts, Manufactures, and Commerce, 439,

The Horticultural Society of London, 665.

The Managers of the Royal Institution, 682.

The Right Honourable Sir Joseph Banks, Bart. K.B. H.M.L.S. 683. 690.

Anton. Bivona Bernardi, 671.

Le Comte de Bournon, F.M.L.S. 672.

Mr. Robert Brown, Libr. L.S. 673. 674. 711.

Alexander P. Buchan, M.D. F.L.S. 677.

Rev. John Burrell, M.A. F.L.S. 688.

Mr. Bracy Clark, F.L.S. 675.

Mr. George Crosfield, 676.

F. Delaroche, M.D. 678.679 .680$.

Lewis Weston Dillwyn, Esq. F.L.S. 538.

The late Jonas Dryander, Esq. V.P.L.S. 681. 706. 707. 708. 709. 710.

Thomas Forster, Esq. F.L.S. 717. 
Adrian Hardy Haworth, Esq. F.L.S. 548.

Sir Thomas Maynard.Hesilrige, Bart. F.L.S. 697.

Aylmer Bourke Lambert, Esq. V.P.L.S. 670. 692.

William Lewis, Esq. F.L.S. 716.

Mr. John Loudon, F.L.S. 685.

Alexander MacLeay, Esq. Sec. L.S. 650.

William Marsden, Esq. F.R.S. 686.

The late Mr. William Martin, F.L.S. 687.

George Montagu, Esq. F.L.S. 561.

Adam Neale, M.D. F.L.S. 689.

David Pennant, Esq. F.L.S. 691.

Henry Reeve, M.D. F.L.S. 694.

Andreas Johannes Retzius, F.M.L.S. 695. 698.

George Shaw, M.D. F.L.S. 511. 698.

John Sims, M.D. F.L.S. 554.

James Edward Smith, M.D. P.L.S. 693. 699. 700.

Mr. James Sowerby, F.L.S. 377. 386. 568. 701.

William Spence, Esq. F.L.S. 702. 703. 704.

Kurt Sprengel, M.D. 684.

John Vaughan Thompson, Esq. F.L.S. 705.

Walter Wade, M.D. A.L.S. 712. 713.714. 


\section{$2 \mathrm{BHL}$ Biodiversity Heritage Library}

1810. "List of Donors to the Library of the Limean Society." Transactions of the Linnean Society of London 10, 411-412.

https://doi.org/10.1111/j.1096-3642.1810.tb00033.x.

View This Item Online: https://www.biodiversitylibrary.org/item/46585

DOI: https://doi.org/10.1111/j.1096-3642.1810.tb00033.x

Permalink: https://www.biodiversitylibrary.org/partpdf/28032

\section{Holding Institution}

Natural History Museum Library, London

\section{Sponsored by}

Natural History Museum Library, London

\section{Copyright \& Reuse}

Copyright Status: Public domain. The BHL considers that this work is no longer under copyright protection.

This document was created from content at the Biodiversity Heritage Library, the world's largest open access digital library for biodiversity literature and archives. Visit BHL at https://www.biodiversitylibrary.org. 